\title{
Multiple-Frequency Investigations of Radio Wave Absorption During the Dawn-Breakup Phase of Auroras
}

\author{
R. Parthasarathy and F. T. Berkey
}

\author{
Contribution From the Geophysical Institute, University of Alaska, College, Alaska
}

(Received July 22, 1964; revised November 5, 1964)

\begin{abstract}
Multiple-frequency and multiple station radio wave absorption data from the auroral zone are used to investigate the electron density content in the $D$ region during a certain type of absorption events. The type is characterized by smooth variability and widespread geographic extent; the approximate electron density profiles are derived and discussed. The general features of the profiles were found compatible with the ionization due to the high energy electrons and the associated bremsstrahlung x-rays. Such events are also discussed in relation to the visual auroral and magnetic records.
\end{abstract}

\section{Introduction}

One of the more significant questions engaging the attention of the radio-physicists in the auroral zone concerns the depth and extent of the radio wave absorbing region. Among the recent outgrowths of the earlier single frequency observations (about $30 \mathrm{Mc} / \mathrm{s}$ ) by Little and Leinbach [1958], and Chapman and Little [1957] is the introduction of the multiple-frequency technique jointly by the CRPL and the Geophysical Institute [Parthasarathy, Lerfald, and Little 1963; Little, Lerfald, and Parthasarathy, 1964]. This paper will seek to present some of the results of the multiplefrequency and multiple-station program, relevant to the associated radio wave absorption during the dawn-breakup phase of the auroral displays.

\section{Method of Deriving $N_{e}(h)$ Profiles}

The method of deriving the ionization profile was discussed in some detail by Parthasarathy et al. [1963], and will only be outlined and supplemented here. The radio wave absorption from any ionized stratum is a function of the wave frequency, the electron gyro frequency, and in the case of the nondeviative absorption, is also proportional to the electron density. For a very narrow beam, zenith directed antenna,

$$
A(f)=\int_{0}^{\infty} K\left(f, \nu_{m}, s, \phi\right) \cdot N_{e}(h) d h
$$

where the absorption as measured by the antenna is $A$, the $K$ function is absorption per unit path length when the electron density is unity, and $N$ is the electron density at height $h$.
The specific absorption $K$ is a function of the electron collision frequency, $\nu_{m}$ (appropriate to the electrons with the most probable velocity), the wave frequency, $f$, the angle of propagation, $\phi$, with respect to the magnetic field, and the electron gyrofrequency, $s$. The form of this function used in the present work is due to Sen and Wyller [1960], which takes into account the dependence of the collision frequency of each electron on its energy [Pack and Phelps, 1961; Phelps 1960]. The function shown in figure 1 , for a set of radio frequencies, is from a tabulation by Jean Chorbajian et al. [1962], by considering the tabulation merely as one of $K\left(f, \nu_{m}, s, \phi\right)$. The collision frequency $\nu_{m}(h)$ required to convert $K\left(f, \nu_{m}, s, \phi\right)$ into $K(f, h)$ was obtained by combining the momentum transfer cross section of Pack and Phelps and the atmospheric gas pressure obtained by rocket data [see, Nordburg, 1961]. The simple relationship is $\nu_{m}=6.3 \times 10^{4} P$, where $P$ is gas pressure in dynes per $\mathrm{cm}^{2}$.

Under certain favorable conditions revealed by the frequency dependence of absorption, a given $N(h)$ function may be tested through the integral relationship (1) to see whether it satisfied the observed data in those frequencies. The larger the frequency range of simultaneous data that is available, the more confidence can one place in the detailed validity of the profile. As regards the type of $N(h)$ profiles that need be considered, the following observational and theoretical surmises may be pointed out. The differential energy spectrum of the downflowing flux, whether protons or electrons, is expressible by a simple power, or exponential law; the atmospheric gas density as well as the effective loss coefficient of electrons by eventual recombination monotonically decrease with height. Under the circumstances, the resulting elec- 
tron density profile may be reasonably expected to approximate a low degree polynomial of height. (That a low degree polynomial is a satisfactory representation of the actual ionization profile may be seen by reference to the proton-derived profiles of Webber and Freier [1963] and the electron- and x-ray-derived profiles of Aikin and Maier [1963].) Basically, the profile derivation outlined in Parthasarathy et al [1963], is only an application of this; the profile was derived by assuming it to be a low degree polynomial (cubic) and then computing the coefficients of the polynomial that could best fit the absorption data.

A feature of the auroral latitudes is the seasonal variation of the gas density and pressure in the $D$ region, which is shown in figure 1 . Because of the trivial variability of $s$ and $\phi$ with height in the zenith $D$ region, it is easy to revise the $K(h)$ obtained from a given $\nu_{m}(h)$, in order to account for the seasonal variation in $\nu_{m}(h)$.

\section{Experimental Details}

Cosmic noise level was monitored at College, Alaska, in eight effective frequencies of $5^{-}, 5^{+}, 10^{-}, 10^{+}, 20^{-}$, $20^{+}, 30$, and $50 \mathrm{Mc} / \mathrm{s}$, where the + and - signs designate the ordinary and extraordinary modes, respectively. The linearly polarized antennas consisted of a pair of $0.4 \lambda$ dipoles spaced $0.4 \lambda$ apart and $0.15 \lambda$ above a reflecting mesh of $1.25 \lambda^{2}$. The beam thus had rotational symmetry about the zenith of $60^{\circ}$ between half power points. The circularly polarized antennas had the form of a square consisting of four $0.4 \lambda$ dipoles, the other details being the same as the linear system. Conventional ring circuits enabled the channeling of the right circular and left circular noise intensities into separate channels, with the appropriate minimum level detectors to reject the atmospherics. For additional experimental details, reference may be made to Parthasarathy and Lerfald [1965].

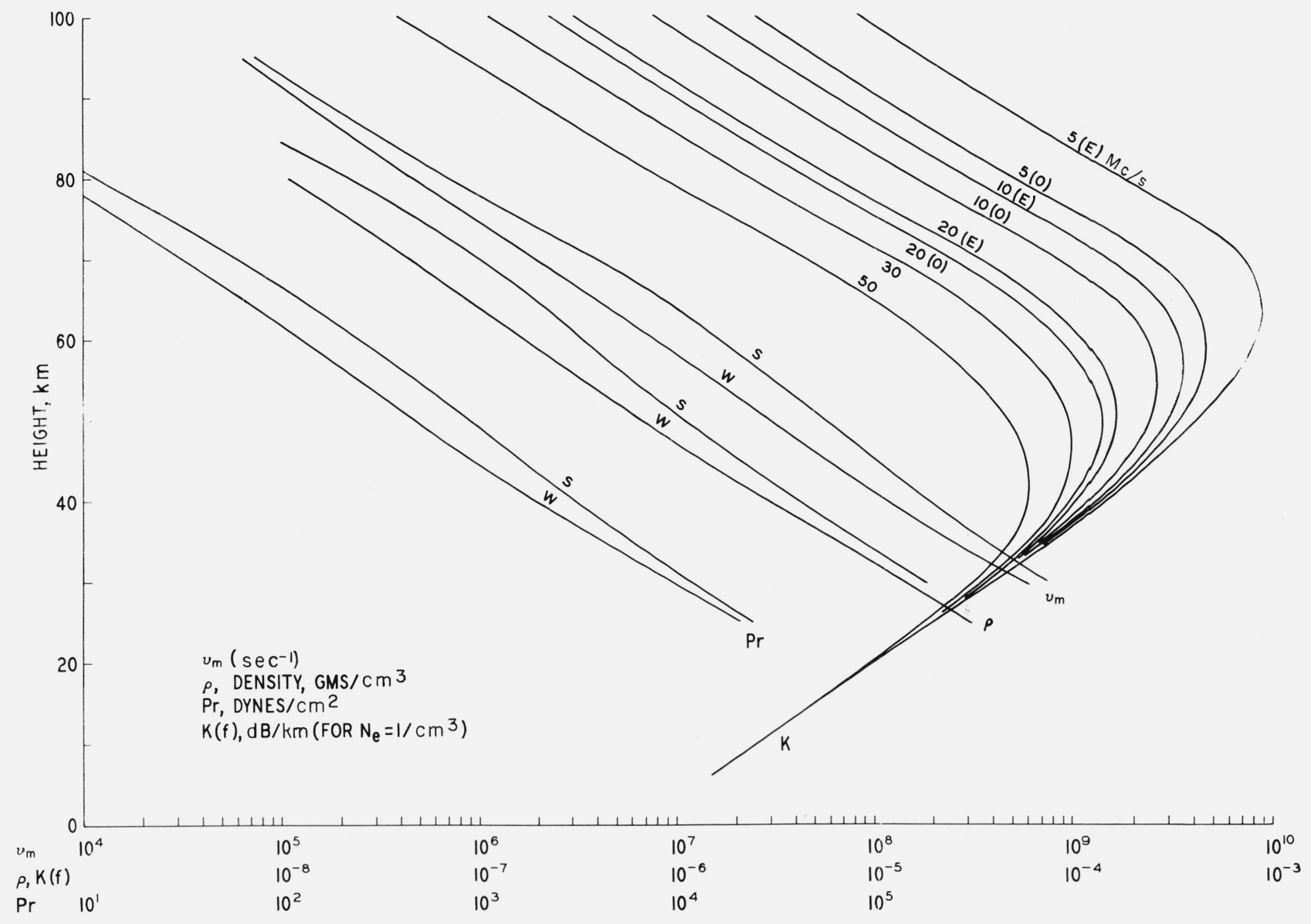

FIGURE 1. Upper atmospheric pressure and density for the summer and winter seasons (S and W) for the auroral zone, after CIRA, 1961. The electron-neutral gas collision frequency $\nu_{m}$ for the two seasons was obtained by combining the gas pressure data and the momentum transfer cross section of Pack and Phelps [1961]. The specific absorption $K(f, h)$ is appropriate to the summer conditions; shifting the curves downwards by $5 \mathrm{~km}$ gives a close enough approximation for the winter $K$ function. 
The multiple station network consisted of $27.6 \mathrm{Mc} / \mathrm{s}$ cosmic noise receivers at College, Ft. Yukon, Kotzebue, and Healy, Alaska.

\section{Data Analysis}

The quiet day levels for the dual polarized frequencies were obtained as detailed by Little et al., [1964]. Essentially, it consists of deriving the absorption-free noise level at any sidereal hour from the fact that, if the absorption were zero, the two circular modes would register equal levels. This dual polarization method of deriving the true level may be considered as one of the more promising techniques available for HF absorption studies. The conventional technique of plotting the levels (from a full year of data) against sidereal time and then drawing the top envelope was used in the case of the linearly polarized frequencies. The raw, wide beam absorption data were then reduced to the equivalent pencil beam data. The reduction is straightforward, providing the distribution of ionization over the major part of the beam could be verified to be approximately uniform.

Auroral absorption events obtained from the Alaskan network of stations were recently analyzed by Berkey and Parthasarathy [1964] who found it possible to group them into a few distinct types. Events which appear isolated and show smooth and slow variability were designated as Type $S$ events. Typical examples may be seen in figures $3 \mathrm{a}$ and $3 \mathrm{c}$. They occur mostly during predawn hours (fig. 2) and invariably in the midst of a few hours of little or no absorption. No seasonal trend was evident during at least the period 1961-1963. They were decidedly more common in the southern boundary (College) of the auroral zone than in the midauroral station of Ft. Yukon.

Comparison with the magnetograms showed that these Type $S$ events could not always be associated with a magnetic bay; and whenever they were associated, it was found that the magnetic bay always preceded by several minutes. There was poor correlation between the magnitudes of the absorption event and the magnetic bay.

Comparison with the all-sky camera pictures showed that the Type $S$ events occurred mostly during the breakup phase of the arcs in the dawn meridians. Typically, the arc becomes progressively brighter, moves slightly equatorward and then breaks up without violent motion; the resulting cloudlike patches, together with the striking feature of a dark, well-bounded $\Omega$-shaped band near the northern side, then move eastward. This phase of the auroral display has been investigated in more detail by Akasofu [1964]. Of relevance to the mechanism of precipitation would be the fact that the histogram of the number of cases versus magnitude of absorption (in steps of $0.5 \mathrm{~dB}$ ) showed a distinct peak at around 1.5 to $2.0 \mathrm{~dB}$ at 30 $\mathrm{Mc} / \mathrm{s}$. For greater details of the analytical results reference may be made to Berkey and Parthasarathy [1964].
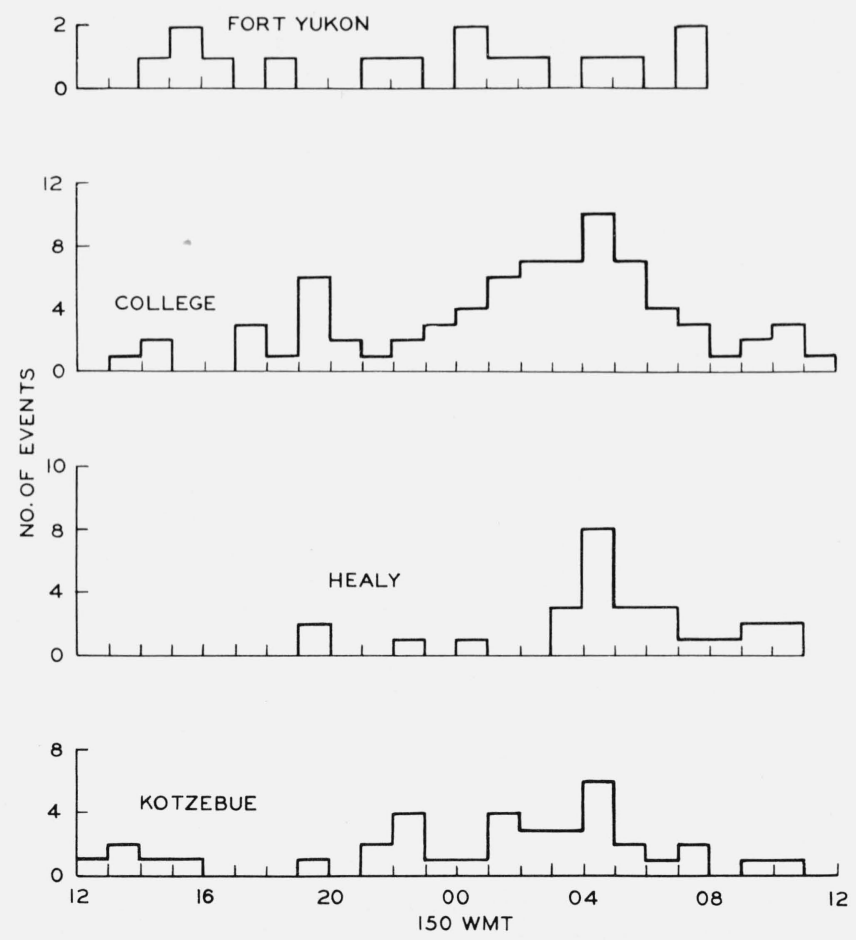

FIGURE 2. Histogram of occurrence frequency of Type $\mathrm{S}$ events in the four Alaskan stations during 1961-1963.

The observations at College and Ft. Yukon extended to 30 months; Kotzebue, 18 months; and Healy, 9 months. 


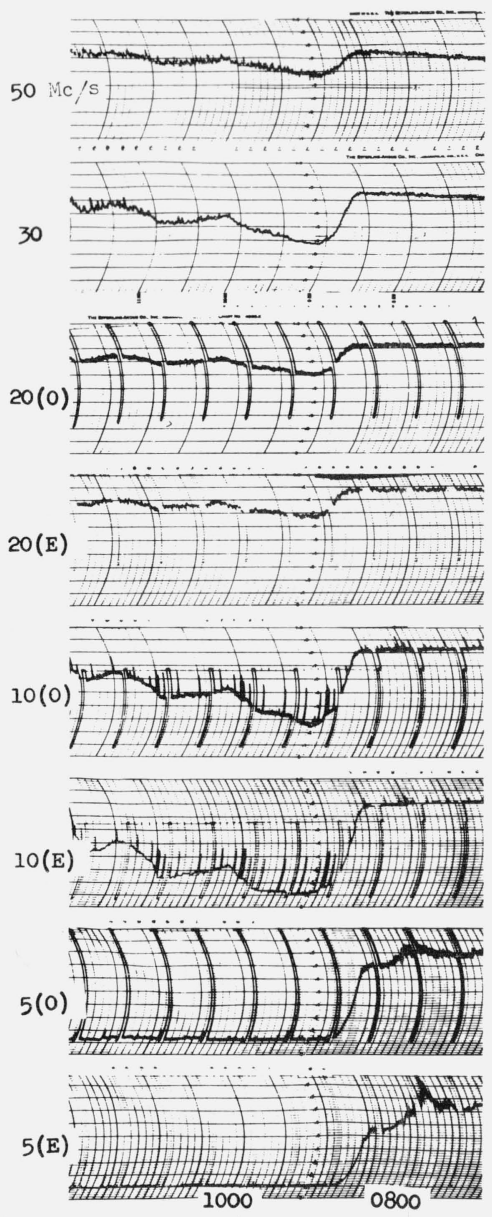

(a)
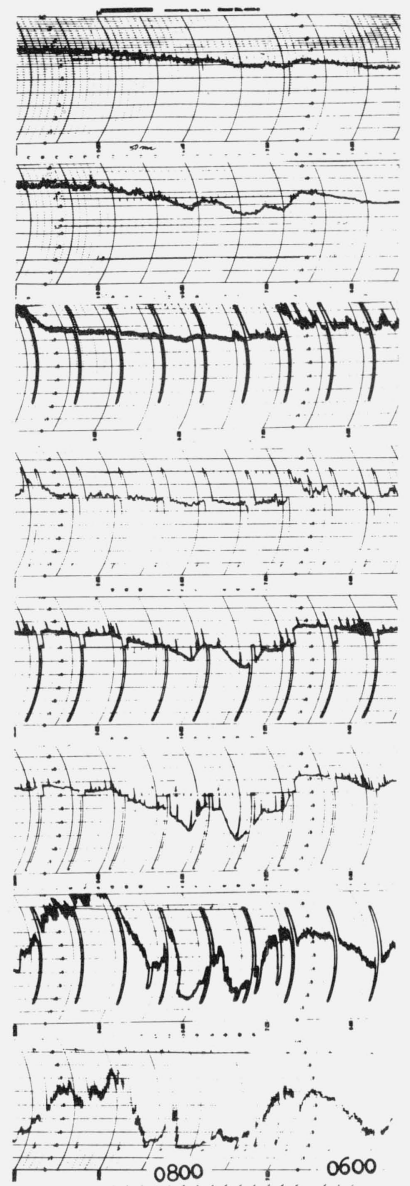

(b)
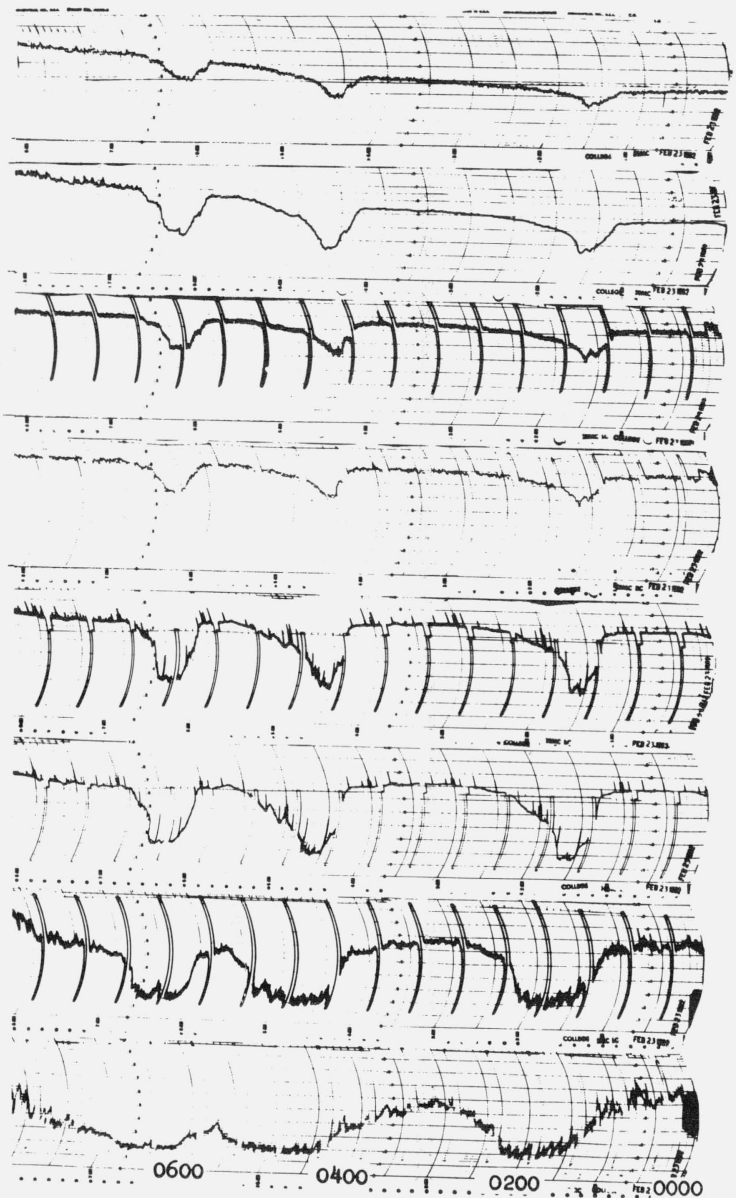

(c)

FIGURE 3. The multiple frequency charts corresponding to the samples of derived electron density profiles shown in Figure 6.

Note the typical shielding action of the $F$ layer during the daytime leading to serious apparent absorption in the lowest of the frequencies, $5-$ and $5+$ Mc/s. Absorption above about $30 \mathrm{~dB}$ cannot be accurately measured in these frequencies. Within the dynamic range, the charts are $\mathrm{dB}$-linear, except the $30 \mathrm{and} 50 \mathrm{Mc} / \mathrm{s}$ channels, which are power linear. Obvious symptoms of interference may also be recognized in some of the charts.

Figure 3 shows several examples of these auroral absorption events as registered in the eight effective frequencies. The geographical extent of the events and hence the approximate uniformity of ionization may be seen in figure 4 which shows the $27.6 \mathrm{Mc} / \mathrm{s}$ absorption in College, Ft. Yukon, and Kotzebue, the latter two being approximately $200 \mathrm{~km}$ (north) and 700 $\mathrm{km}$ (west) respectively. No data from stations south of College were available for the events in figure 4, but whenever data for similar events were available from Healy (100 km to the south), only minor differences would be noticed between the absorption at College and at Healy.

The reduced pencil beam data for a few instants are plotted in figure 5 . Using the values of absorption read on the best fitting line for the six or eight effective frequencies (rather than the acutal reduced data), the instantaneous ionization profiles shown in figure 6 were then derived. It may be emphasized
[Paratharathy et al., 1963] that the profiles tend to be somewhat uncertain beyond about $80 \mathrm{~km}$. However, by multiplying the $N(h)$ profiles with the winter $K$ functions for any of the frequencies between 5 and 50 $\mathrm{Mc} / \mathrm{s}$, it may be seen that the observed absorption datum in each of the frequencies is fully ( $\geq 95 \%)$ accountable by the profiles in the region below the $85 \mathrm{~km}$ stratum. Arbitrary models of alternate profiles which do not ensure a dominant absorbing region below 85 $\mathrm{km}$ would be incapable of yielding the observed frequency dependence of absorption in figure 5 . The exact shape of the profile in the region contributing only a minor fraction to the data $(\gtrsim 80 \mathrm{~km})$ is open to arbitrary adjustments, subject only to the requirements of physically plausible shapes, and the integrated absorption in the adjustable region remains small. Because of their sensitivity to regions above $80 \mathrm{~km}$, the need for data below $5 \mathrm{Mc} / \mathrm{s}$ to supplement the $\mathrm{HF}$ data can hardly be overemphasized in ensuring more accurate higher altitude profiles. 

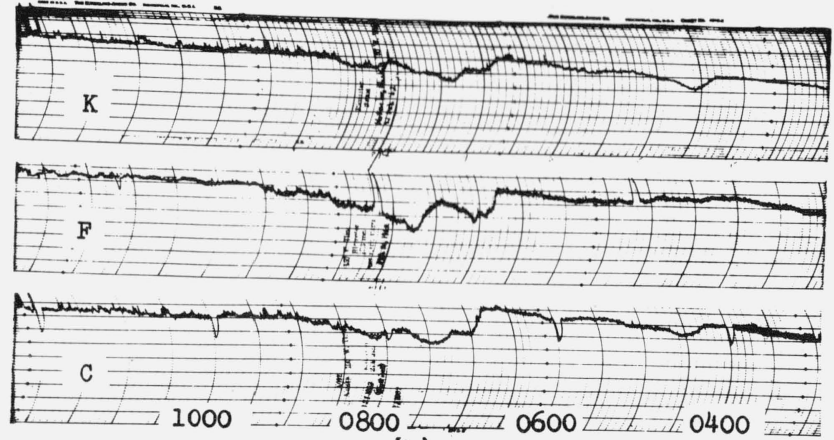

(b)
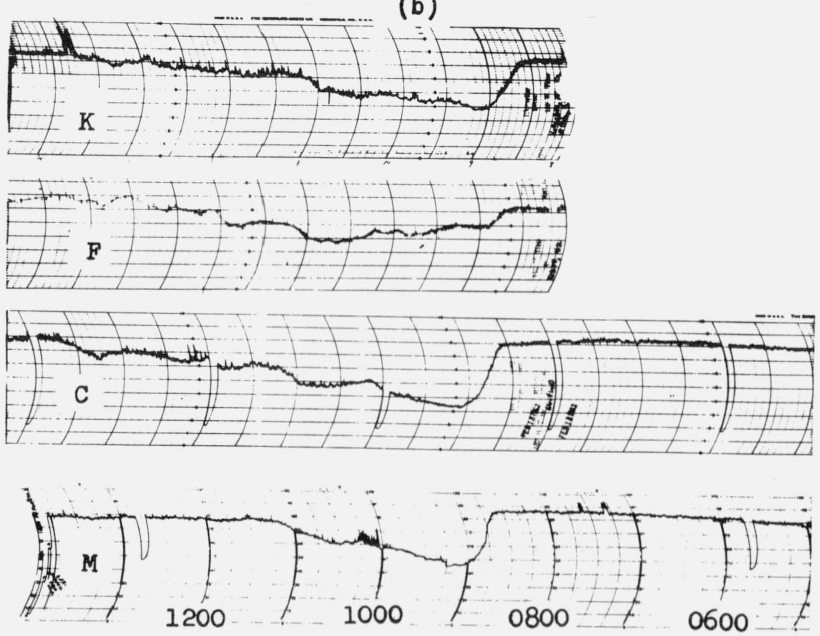

(a)

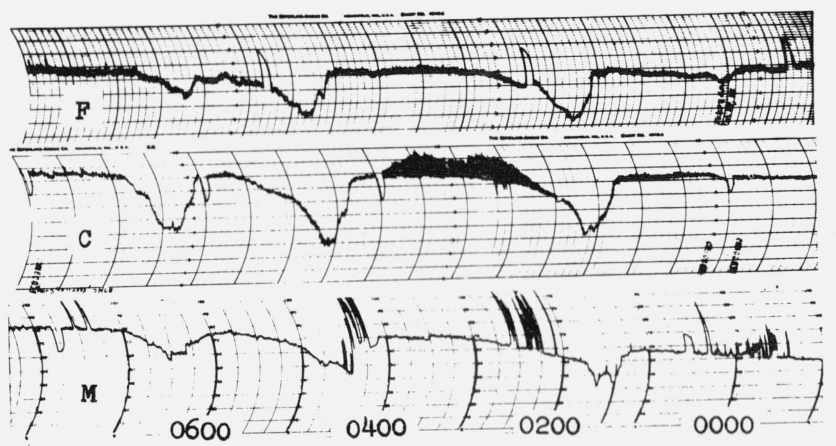

(c)
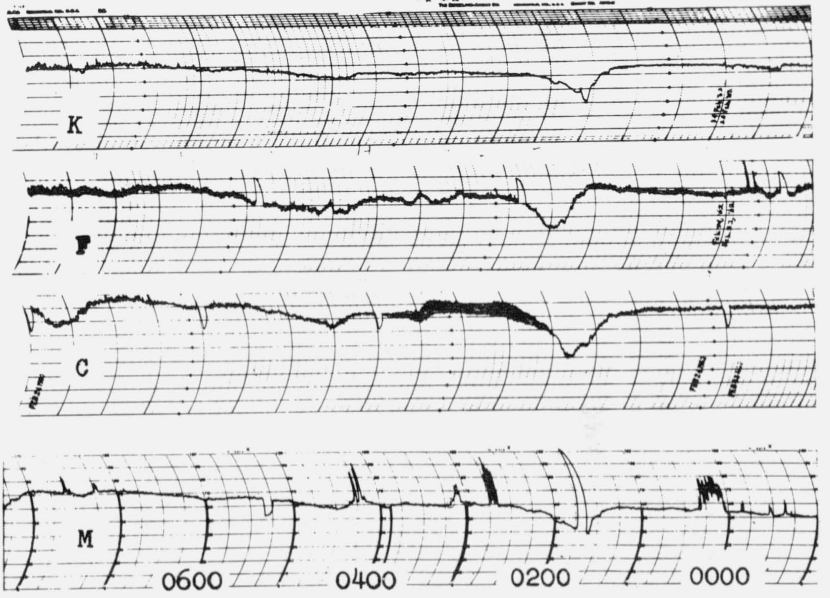

(d)

Figure 4. The $27.6 \mathrm{Mc} / \mathrm{s}$ records from the other Alaskan stations of Ft. Yukon (F), College (C), Kotzebue (30 Mc/s) (K), and the conjugate area station in Macquarie Island $(m) ;(a),(b)$, and $(c)$ are during the times of the corresponding ones in figure 3.

\section{Discussion of Results}

A few obvious inferences may be made from the profiles, appropriate to these isolated, smooth, and geographically widespread absorption events. Profiles which extend down to these low altitudes seem compatible with the ionization due to the electrons and the associated x-ray Bremsstrahlung, such as those proposed by Chapman and Little [1957] and Aikin and Maier [1963]. Ansari [1963] has pointed out that electrons of $10 \mathrm{keV}$ energy are inefficient sources of x-rays; detailed calculation of $\mathrm{x}$-ray production rates has also been made by Rees [1964]. It was not found possible, however, to propose from among these (and the many other computed profiles) a 'model' profile. A statistical analysis, using a limited amount of data (from 25 Type $S$ events), showed that the events may be defined in the frequency range of 5 to $50 \mathrm{Mc} / \mathrm{s}$ by the relation $\mathrm{A}(f)=K \cdot f^{-n}$. The values of $n$ ranged between 1.3 and 1.9 , with a mean of 1.5. This mean value may be contrasted with the value of 1.8 when absorption events were considered, without classifying them into the several types [Lerfald et al., 1964]. The obvious inference can thus be made that during the dawn-breakup phase of the aurora, the primary particle spectrum is typically much harder than at other times. Of major significance would be that during any auroral display, and decidedly so during the breakup phase, the region relevant to HF-VHF absorption is well below the visual auroral altitude of about $100 \mathrm{~km}$ and extends down to the $50 \mathrm{~km}$ region. An essentially similar inference was earlier made by Little and Leinbach [1958] by comparing the $f$-min data and the simultaneous cosmic noise absorption data at $30 \mathrm{Mc} / \mathrm{s}$.

Parthasarathy and Berkey [1965] had also found that the frequency exponent during the Type $F$ events was, on an average, again about 1.5. The Type $F$ events consist of those with a fast, flash-like onset of absorption and were found to be typical of the midnight breakup phase of quiet auroral forms.

The $N_{e}(h)$ profiles may be used to derive the energy spectrum of the primary electrons if the rate coefficients of recombination, attachment, etc., are known as a function of height. The effective recombination coefficient, $\alpha_{\text {eff }}(h)$, may be derived by using the basic laboratory data in conjunction with the atmospheric parameters [see, for example, Webber, 1962]. The 

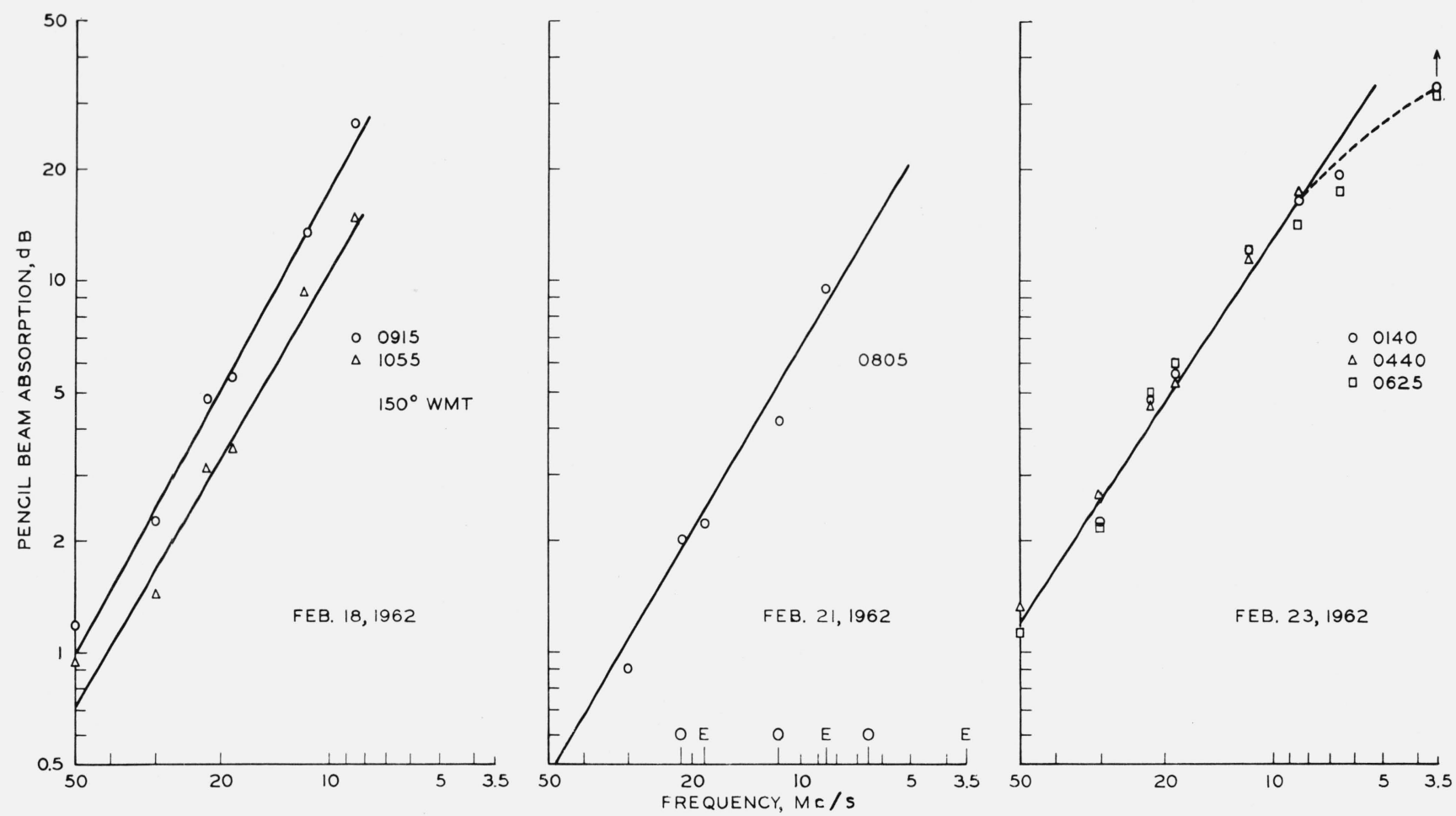

FIGURE 5. The reduced pencil beam absorption versus frequency.

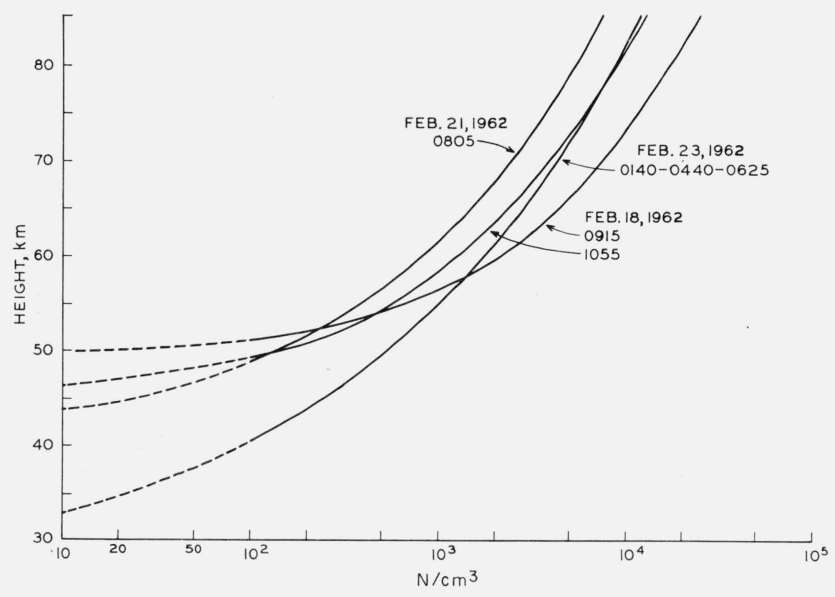

FIGURE 6. Electron density profiles derived by using the observed radiowave absorption data from figure 5 and the winter parameters in figure 1 (see text).

alternative approaches (to be published) would be to derive the coefficient by two independent methods: (a) By means of a set of polar cap event (PCE) electron density profiles, and the corresponding satellite measured proton flux data; and (b) by means of the $N_{e}(h)$ profiles obtained during the recovery phase of a special category of absorption events characterized by their flash-like onset and rapid self-recovery.
The multiple frequency program and its application to profile derivation are due to the very close collaboration between one of us (R. P) and C. G. Little and G. M. Lerfald of the CRPL. We acknowledge the enthusiastic help of Lillian Bogert and D. Ruff in the scaling and data reduction processes. The investigation was financed partly by the Advanced Research Projects Agency under Contract No. 186-61 and partly by the National Science Foundation under Grant GP-947.

\section{References}

Aikin, A. C., and E. J. Maier (1963), The effect of auroral Bremsstrahlung on the lower ionosphere, Technical Report NASA TN D-2096, NASA.

Akasofu, S. I. (1964), The development of the auroral substorm, Planetary Space Sci. 12, No. 4, 273-282.

Ansari, Z. A. (1963), The spatial and temporal variations in high latitude cosmic noise absorption and their relation to luminous aurora, University of Alaska Geophysical Institute Scientific Report UAG-R138.

Berkey, F. T., and R. Parthasarathy (1964), An investigation of selected types of radiowave absorption events in the auroral zone, University of Alaska, Geophysical Institute Scientific Report UAG-R151.

Chapman, S., and C. G. Little (1957), The non-deviate absorption of high frequency radiowaves in auroral latitudes, J. Atmospheric Terrest. Phys. 10, 20.

Chorbajian, J. J., M. Sugiura, and R. Parthasarathy (1962), Radiowave absorption coefficients based on Sen-Wyller magneto-ionic formula, University of Alaska, Geophysical Institute Report No. 9. 
Lerfald, G. M., C. G. Little, and R. Parthasarathy (1964), A study of D-region electron density profiles during auroras, using the multiple frequency cosmic noise technique, J. Geophys. Res. 69, No. $13,2857-2860$.

Little, C. G., and H. Leinbach (1958), Some measurements of high latitude ionosphere absorption using extra-terrestrial radio waves, Proc. IRE 46, 334-348.

Little, C. G., G. M. Lerfald, and R. Parthasarathy (1964), The extension of cosmic noise absorption measurements to lower frequencies using polarized antennas, Radio Sci. J. Res. NBS/USNCURSI 68D, No. 8, 859-865.

Nordberg, W. (1961), Density and pressure data, in CIRA 1961, COSPAR International Reference Atmosphere, p. 56 (NorthHolland Publishing Company, Amsterdam).

Pack, J. L., and A. V. Phelps (1961), Drift velocities of slow electrons in helium, neon, argon, hydrogen, and nitrogen, Phys. Rev. $121,798$.

Parthasarathy, R., G. M. Lerfald, and C. G. Little (1963), Deviation of electrondensity profiles in the lower ionosphere, using radio absorption measurements at multiple frequencies, J. Geophys. Res. 68, No. 12, 3581-3588.

Parthasarathy, R., and G. M. Lerfald (1965), Cosmic noise survey at 65 degrees $(\mathrm{N})$ declination in the $5-50 \mathrm{Mc} / \mathrm{s}$ band, Monthly Notices RAS (in press).
Parthasarathy, R., and F. T. Berkey (1965), Auroral zone studies of sudden-onset radiowave absorption events using multiple-frequency data, J. Geophys. Res. 70, No. 1.

Phelps, A. V. (1960), Propagation constants for electromagnetic waves in weakly ionized, dry air, J. Appl. Phys. 31, 1723.

Rees, M. H. (1964), Ionization in the earth's atmosphere by aurorally associated bremsstrahlung x-rays, Planetary Space Sci. 12, No. $11,1093-1108$.

Sen, H. K., and A. A. Wyller (1960), On the generalization of the Appleton-Hartree magnetoionic formulas, J. Geophys. Res. 65, No. 12, 3931-3950.

Webber, W. R. (1962), The production of free electrons in; the ionospheric $D$-layer by solar and galactic cosmic rays and the resultant absorption of radiowaves, J. Geophys. Res. 67, 5091.

Webber, W. R., and P. S. Freier (1963), Electron density profiles in the lower ionosphere due to solar cosmic rays, J. Geophys. Res. 68, 6223.

(Paper 69D3-479) 